\title{
Differential effects on metabolic parameters between sitagliptin and alogliptin in drug naïve subjects with type 2 diabetes
}

\author{
Eiji Kutoh ${ }^{1,2^{*}}$ and Hiroshi Yamashita ${ }^{1}$ \\ Correspondence: ekuto@excite.com \\ 'Biomedical Center, Tokyo, Japan. \\ 2Division of Diabetes and Endocrinology, Department of Internal Medicine, Gyoda General Hospital, Saitama, Japan.
}

\begin{abstract}
Background: Different dipeptidyl peptidase (DPP)-4 inhibitors show significant structural differences. The aim of this study was to find any differential effects on metabolic parameters between sitagliptin and alogliptin.

Methods: Drug naive subjects with type 2 diabetes (T2DM) were assigned to either $25-100 \mathrm{mg} /$ day sitagliptin $(\mathrm{n}=37)$ or $6.25-25 \mathrm{mg} /$ day alogliptin $(\mathrm{n}=35)$ monotherapy. At 3 months, the levels of glycemic and non-glycemic parameters were compared with those at baseline.

$\underline{\text { Results: }}$ At baseline, the levels of these parameters were similar between these two groups. At 3 months, similar glucose lowering efficacies were observed in both groups. However, distinct regulatory patters were observed with lipid profiles. In the sitagliptin group, high triglyceride (TG) and free fatty acid (FFA) levels significantly decreased. However, in the alogliptin group, significant reductions of total cholesterol (T-C), non-high density lipoprotein (non-HDL)-C and low density lipoprotein (LDL)-C levels were observed and changes of $(\Delta) \mathrm{HbA} 1 \mathrm{c}$ levels were weakly correlated with those of $\Delta \mathrm{T}-\mathrm{C}, \Delta$ non-HDL-C or $\triangle \mathrm{LDL}-\mathrm{C}$. Besides these lipid parameters, homeostasis model assessment (HOMA)-B increased in both groups $(+52.3 \%$ for sitagliptin and $+91.2 \%$ for alogliptin) with significant inter-group differences $(\mathrm{p}<0.05)$. Both drugs are well tolerated and no clinically significant adverse events were observed. However, uric acid (UA) levels increased, although still within normal limit, in both groups.

Conclusions: This study suggests that: 1) both sitagliptin and alogliptin are effective and safe as an initial therapy for T2DM. 2) alogliptin can ameliorate atherogenic lipid profiles and these atherogenic lipids may be associated with the glycemic effect during alogliptin treatment. By contrast, sitagliptin can down-regulate high TG and FFA levels. 3) although similar glucose lowering efficacies were observed, sitagliptin had a higher degree of enhancing beta-cell function than alogliptin.
\end{abstract}

Keywords: Incretin based therapy, type 2 diabetes, DPP-4 inhibitor, sitagliptin, alogliptin

\section{Introduction}

Incretin-based drugs, glucagons like peptide-1 (GLP-1) receptor agonists and DPP-4 inhibitors represent a new therapeutic approach for T2DM $[\mathbf{1}, \mathbf{2}, 3]$. Since incretinbased therapies enhance beta-cell function, they may be an appropriate therapeutic option early in the disease when the patients still maintain sufficient levels of betacell function [4]. However, to date, no evidence is available whether these drugs can be used as a first-line option for T2DM.

At the present time, at least six DPP-4 inhibitors are clinically available on the market (sitagliptin, vildagliptin, saxagliptin, alogliptin, linagliptin and teneligliptin) and many others are coming soon. Although these drugs all inhibit the same enzyme (DPP-4) and display similar glucose lowering efficacies, they show clearly distinct chemical structures $[5,6]$. This fact implicates that these drugs may have different pharmacological properties, such as half-life, systemic exposure, bioavailability, protein binding, metabolism, presence of active metabolites and excretion routes $[6,7]$. Data on non-glycemic parameters are scant at present and do not allow a comparison among DPP-4 inhibitors. Thus it is of therapeutic value to analyze the differences in glycemic and non-glycemic efficacies of the different DPP-4 inhibitors. To undertake such studies, it makes sense to perform with drug naïve subjects as monotherapy in order to eliminate the influences of other drugs as much as possible.

Sitagliptin is the first DPP- 4 inhibitor on the market and is the most widely used drug of this class throughout the world. Its efficacy and safety are extensively studied and well acknowledged $[8,9,10]$. On the contrary, alogliptin is a quinazolinone-based, non-covalent DPP-4 inhibitor currently available only in Japan and in advanced clinical

(C) 2012 Kutoh et al; licensee Herbert Publications Ltd. This is an open access article distributed under the terms of Creative Commons Attribution License (http://creativecommons.org/licenses/by/3.0),This permits unrestricted use, distribution, and reproduction in any medium, provided the original work is properly cited. 
phases in other countries including the USA and EU $[11,12,13]$. The reductions in $\mathrm{HbA1c}$ levels with alogliptin appear to be in the similar range as with the other DPP-4 inhibitors $[4,14,15]$. However, due to its limited availability (only in Japan) its effects are largely unknown in actual clinical settings. Overall, DPP-inhibitors are regarded as "lipid neutral" [16]. However, it was preliminarily shown that alogliptin has a favorable effect in down-regulating atherogenic lipid profiles and post-prandial lipid $[4,17]$.

As an initial step towards investigating the differences between different DPP-4 inhibitors, comparison between sitagliptin and alogliptin on metabolic parameters was performed with drug naïve subjects of T2DM.

\section{Subjects and methods \\ Subjects}

Inclusion criteria were those who were newly diagnosed with type 2 diabetes (T2DM) or those who were previously diagnosed but were untreated. The diagnosis was made according to the criteria of the Japan Diabetes Society [18]. All the subjects had not received any regularly prescribed drugs in the 6 months prior to the study.

Exclusion criteria were those with clinically significant renal [creatinine (CRE) $>1.5 \mathrm{mg} / \mathrm{dl}$ ], liver [glutamic oxalacetic transaminases/glutamic pyruvic transaminases (GOT/GPT)> 70/70 IU/I], heart [brain natriuretic peptide (BNP) $>70 \mathrm{pg} /$ $\mathrm{ml}$ ], hypertensive [blood pressure above $160 / 100 \mathrm{mmHg}$ ] disorders, type 1 diabetes (T1DM) and pregnancy. These subjects were recruited from the outpatient department of Diabetes and Endocrinology of Gyoda General Hospital (Saitama, Japan) and two other related hospitals. These patients received $25-100 \mathrm{mg} /$ day sitagliptin $(\mathrm{n}=37$ ) or 6.25 $25 \mathrm{mg} /$ day alogliptin monotherapy $(n=35)$. The patients started with the lowest dose of these drugs (sitagliptin $25 \mathrm{mg} /$ day and alogliptin $6.25 \mathrm{mg} /$ day). The doses were increased according to the results of the glycemic control. At the end of the study, most subjects (approximately $90 \%$ in both groups) used the dose of sitagliptin $50 \mathrm{mg} /$ day and alogliptin $12.5 \mathrm{mg} /$ day. They were assigned without strict randomized procedure and the study design was not a blinded one. In both groups, the subjects were encouraged to follow the exercise and diet suggested by the American Diabetes Association [19]. Informed consents were obtained from the patients and the protocol of this study was approved by the Ethical Committee. This study was conducted in accordance with principles of Good Clinical Practice. In the case of unacceptable or undesirable therapeutic outcome, the patients were free to leave the therapy whenever they wished. The drop out subjects were excluded from data analysis.

\section{Laboratory measurements}

The primary end point was the changes in HbA1c levels from baseline to 3 months. The HbA1c values were assessed by the JDS standardization [20] throughout this manuscript. To convert these into the National Glycoprotein Standardization Program (NGSP), add approximately $0.4 \%$ to the indicated values [20]. Patients with $\geq 1 \%$ reduction in $\mathrm{HbA1c}$ at 3 months were designated as responders and those with $<1 \%$ reduction were designated as non-responders. This $1 \%$ cut off value has been traditionally used in other studies [21].

The secondary end point included fasting blood glucose (FBG), and other metabolic parameters including $\mathrm{T}-\mathrm{C}, \mathrm{TG}$, HDL-C, LDL-C, UA, HOMA-R, HOMA-B and body mass index (BMI).

Blood was collected at the fasting state before breakfast and the standard technique was used to measure these parameters as described previously [21]. Measurements of $\mathrm{HbA1c}$ and FBG (measured by the system form Arkray, Shiga, Japan) were performed once a month. Insulin (measured by the kit from Abbott Japan, Tokyo), T-C, TG, HDL-C, nonHDL-C and LDL-C (measured by the kit from Nittobo, Tokyo, using Hitachi 7180 analyzer) was measured at the start (baseline) and at the end (3 months) of the study. Antiglutamic acid decarboxylase (GAD) antibody was measured in some suspected patients in order to exclude those with T1DM (Mitsubishi BML, Tokyo, Japan). HOMA-R and HOMA-B were calculated as described [22]; $\mathrm{HOMA}-\mathrm{R}=$ insulin $(\mu \mathrm{U} / \mathrm{mL}) \times \mathrm{FBG}(\mathrm{mg} / \mathrm{dL}) / 405$, HOMA-B=insulin $(\mu \mathrm{U} / \mathrm{mL}) \times 360 /$ FBG $(\mathrm{mg} / \mathrm{dL})-63$. Liver (GOT, GPT, alkaline phosphatase: ALP, and gamma-glutamyl transpeptidase $: \gamma-G T P$ ) and renal (BUN and CRE) functions were also monitored monthly. In the case of any significant increases of these parameters, administration of sitagliptin or alogliptin was planned to discontinue. The drop-out subjects were excluded from data analysis.

\section{Data analyses}

Change was calculated as the values at 3 months (posttherapy) minus those at baseline (pre-therapy). Unpaired Student's t-test was employed to analyze the difference at baseline between these two groups (sitagliptin and alogliptin). When the data were normally distributed, paired Student's t-test was used to analyze the changes in each group (intra-group differences). When the data were not normally distributed, Wilcoxon signed-rank test was employed. An analysis of covariance (ANCOVA) was used to analyze the inter-group differences. To identify any factors that influence the efficacy of these drugs, multiple regression analyses between the changes of $\mathrm{HbA1c}$ levels and the baseline levels of other parameters were undertaken. Simple regression analysis was performed to analyze the changes between measured parameters. The results were expressed as the mean \pm SD. Throughout the statistical analysis, values of $p<0.05$ were considered significant.

\section{Results}

Baseline characteristics and changes of the parameters at 3 months.

As shown in table I, the baseline characteristics were 
Kutoh et al. Journal of Diabetes Research \& Clinical Metabolism 2012,

http://www.hoajonline.com/journals/pdf/2050-0866-1-17.pdf

Table I. Baseline characteristics of the subjects in the sitagliptin and alogliptin groups

\begin{tabular}{llll}
\hline & sitagliptin & alogliptin & p-values \\
\hline age $($ years old $)$ & $53.5 \pm 11.5$ & $50.0 \pm 12.9$ & n.s. \\
sex $(\mathrm{F} / \mathrm{M})$ & $7 / 30$ & $4 / 31$ & n.s. \\
FBS $(\mathrm{mg} / \mathrm{dl})$ & $226.1 \pm 64.6$ & $221.4 \pm 53.7$ & n.s. \\
HbA1c $(\%)$ & $10.43 \pm 2.21$ & $10.40 \pm 2.08$ & n.s. \\
T-C $(\mathrm{mg} / \mathrm{dl})$ & $227.1 \pm 42.2$ & $240.8 \pm 40.8$ & n.s. \\
TG $(\mathrm{mg} / \mathrm{dl})$ & $231.6 \pm 222.0$ & $209.0 \pm 220.0$ & n.s. \\
HDL-C $(\mathrm{mg} / \mathrm{dl})$ & $52.4 \pm 12.5$ & $52.4 \pm 17.9$ & n.s. \\
nonHDL-C $(\mathrm{mg} / \mathrm{dl})$ & $174.6 \pm 41.3$ & $188.4 \pm 41.0$ & n.s. \\
LDL-C $(\mathrm{mg} / \mathrm{dl})$ & $147.7 \pm 32.2$ & $155.9 \pm 52.4$ & n.s. \\
FFA $(\mathrm{eEq} / \mathrm{l})$ & $0.957 \pm 0.333$ & $0.808 \pm 0.300$ & n.s. \\
UA $(\mathrm{mg} / \mathrm{dl})$ & $5.11 \pm 1.42$ & $4.81 \pm 1.47$ & n.s. \\
insulin $(\mu \mathrm{U} / \mathrm{ml})$ & $6.86 \pm 4.52$ & $8.03 \pm 6.02$ & n.s. \\
HOMA-R & $3.79 \pm 2.91$ & $4.48 \pm 3.51$ & n.s. \\
HOMA-B & $18.33 \pm 15.59$ & $20.26 \pm 20.00$ & n.s. \\
BMI & $25.22 \pm 4.63$ & $26.51 \pm 5.84$ & n.s. \\
\hline
\end{tabular}

similar and no differences of these parameters (age, gender, FBG, HbA1c, T-C, TG, HDL-C, non-HDL-C, LDL-C, FFA, UA, insulin, HOMA-R, HOMA-B and BMI) were observed between these two groups.

At 3 months, similar, significant reductions of $\mathrm{HbA1c}$ and FBG levels were observed in these two groups (compare Table IIA1 and IIB). 13 out of 37 subjects ( $35.1 \%$ in the sitagliptin group) and 13 out of 35 subjects ( $37.1 \%$ in the alogliptin group) were non-responders whose $\mathrm{HbA} 1$ had less than $(<)$ a $1 \%$ reduction from the baseline.

In an effort to find any predictive parameters for the efficacy of sitagliptin or alogliptin, multiple regression analysis was performed between the changes of $(\Delta) \mathrm{HbA1c}$ levels and the baseline levels of the metabolic parameters including $\mathrm{FBG}, \mathrm{HbA1C}, \mathrm{T}-\mathrm{C}, \mathrm{TG}, \mathrm{HDL}-\mathrm{C}$, non-HDL-C, LDL-C, FFA, UA, insulin, HOMA-R, HOMA-B or BMI. Among these factors, baseline $\mathrm{HbA1c}$ levels were chosen as the significant contributing factor for the glycemic efficacy $(\triangle \mathrm{HbA} 1 \mathrm{c})$ both in the sitagliptin and alogliptin groups. To further consolidate this finding, simple regression analysis was performed between $\triangle \mathrm{HbA1c}$ and baseline $\mathrm{HbA1c}$ levels. As shown in figure 1, significant correlations were observed in both groups ( $R=-0.536$ for sitagliptin, $R=-0.345$ for alogliptin).

\section{Differential effects between sitagliptin and alogliptin} on beta beta-cell function

DPP-4 inhibitors are known to augment beta-cell function, however, their effects on insulin resistance (sensitivity) or body weight remain somewhat elusive. This question has been investigated using HOMA-B (for beta-cell function) and HOMA-R (for insulin resistance) indexes. As shown in Table II A1 and II B, no changes, if any, were observed
Table II Changes of glycemic and other parameters. A1) sitagliptin

\begin{tabular}{llll}
\hline & baseline & 3 month & p-values \\
\hline FBS $(\mathrm{mg} / \mathrm{dl})$ & $226.1 \pm 64.6$ & $190.4 \pm 78.7$ & $<0.002$ \\
$\mathrm{HbAlc}(\%)$ & $10.43 \pm 2.21$ & $8.24 \pm 2.18$ & $<0.00001$ \\
$\mathrm{~T}-\mathrm{C}(\mathrm{mg} / \mathrm{dl})$ & $227.1 \pm 42.2$ & $221.2 \pm 42.3$ & n.s. \\
$\mathrm{TG}(\mathrm{mg} / \mathrm{dl})$ & $231.6 \pm 222.0$ & $195.6 \pm 167.7$ & 0.0651 \\
HDL-C $(\mathrm{mg} / \mathrm{dl})$ & $52.4 \pm 12.5$ & $53.5 \pm 11.5$ & n.s. \\
nonHDL-C $(\mathrm{mg} / \mathrm{dl})$ & $174.6 \pm 41.3$ & $167.6 \pm 41.6$ & n.s. \\
LDL-C $(\mathrm{mg} / \mathrm{dl})$ & $147.7 \pm 32.2$ & $145.9 \pm 35.9$ & n.s. \\
FFA $(\mathrm{eEq} / \mathrm{l})$ & $0.957 \pm 0.333$ & $0.810 \pm 0.334$ & $<0.05$ \\
UA $(\mathrm{mg} / \mathrm{dl})$ & $5.11 \pm 1.42$ & $5.67 \pm 1.35$ & $<0.0005$ \\
insulin $(\mu \mathrm{U} / \mathrm{ml})$ & $6.82 \pm 4.52$ & $8.34 \pm 6.83$ & $<0.005$ \\
HOMA-R & $3.79 \pm 2.91$ & $4.09 \pm 4.54$ & n.s. \\
HOMA-B & $18.33 \pm 15.59$ & $35.05 \pm 42.71$ & $<0.005$ \\
BMI & $25.22 \pm 4.63$ & $25.41 \pm 4.56$ & n.s. \\
\hline
\end{tabular}

Table II A2) TG changes in sitagliptin group

\begin{tabular}{llll}
\hline & baseline & 3 months & p-values \\
\hline $\begin{array}{l}\text { normal TG } \\
(\leq 199 \mathrm{mg} / \mathrm{dl})\end{array}$ & $117.6 \pm 45.1$ & $138.0 \pm 70.4$ & n.s. \\
$\begin{array}{l}\text { high TG } \\
(\geq 200 \mathrm{mg} / \mathrm{dl})\end{array}$ & $469.9 \pm 256.7$ & $315.5 \pm 241.0$ & $<0.001$ \\
\hline
\end{tabular}

Table II B) Alogliptin

\begin{tabular}{llll}
\hline & baseline & 3 month & p-values \\
\hline FBS $(\mathrm{mg} / \mathrm{dl})$ & $221.4 \pm 53.7$ & $192.3 \pm 60.5$ & $<0.02$ \\
HbA1c $(\%)$ & $10.40 \pm 2.08$ & $8.80 \pm 2.32$ & $<0.00001$ \\
T-C $(\mathrm{mg} / \mathrm{dl})$ & $240.8 \pm 40.8$ & $225.7 \pm 38.5$ & $<0.02$ \\
TG $(\mathrm{mg} / \mathrm{dl})$ & $209.0 \pm 220.0$ & $171.9 \pm 131.0$ & n.s. \\
HDL-C $(\mathrm{mg} / \mathrm{dl})$ & $52.4 \pm 17.9$ & $53.2 \pm 13.2$ & n.s. \\
nonHDL-C $(\mathrm{mg} / \mathrm{dl})$ & $188.4 \pm 41.0$ & $172.5 \pm 39.0$ & $<0.005$ \\
LDL-C $(\mathrm{mg} / \mathrm{dl})$ & $155.9 \pm 52.4$ & $145.1 \pm 45.5$ & $<0.05$ \\
FFA $(\mathrm{eEq} / \mathrm{l})$ & $0.808 \pm 0.300$ & $0.722 \pm 0.277$ & n.s. \\
UA $(\mathrm{mg} / \mathrm{dl})$ & $4.81 \pm 1.47$ & $5.45 \pm 1.65$ & $<0.00001$ \\
insulin $(\mu \mathrm{U} / \mathrm{ml})$ & $8.03 \pm 6.02$ & $9.52 \pm 7.27$ & 0.0052 \\
HOMA-R & $4.48 \pm 3.51$ & $4.67 \pm 3.92$ & n.s. \\
HOMA-B & $20.26 \pm 20.00$ & $30.87 \pm 25.35$ & $<0.001$ \\
BMI & $26.51 \pm 5.84$ & $26.52 \pm 5.66$ & n.s. \\
\hline
\end{tabular}


A: sitagliptin

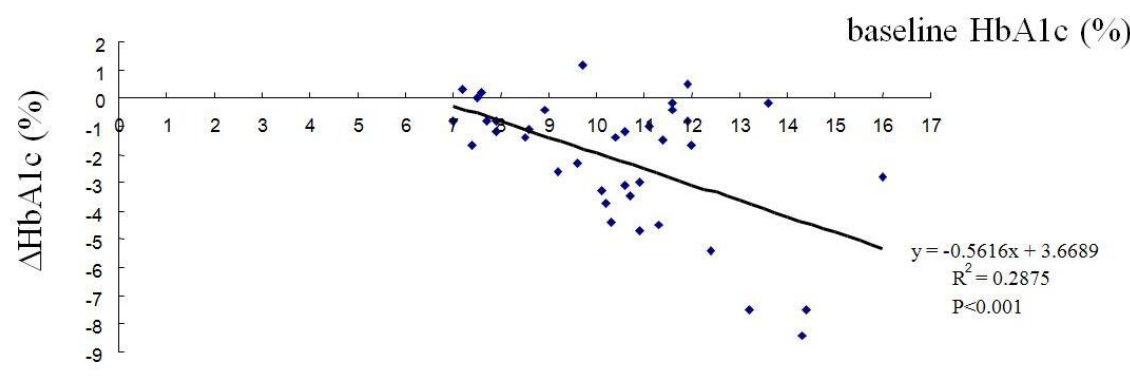

B: alogliptin

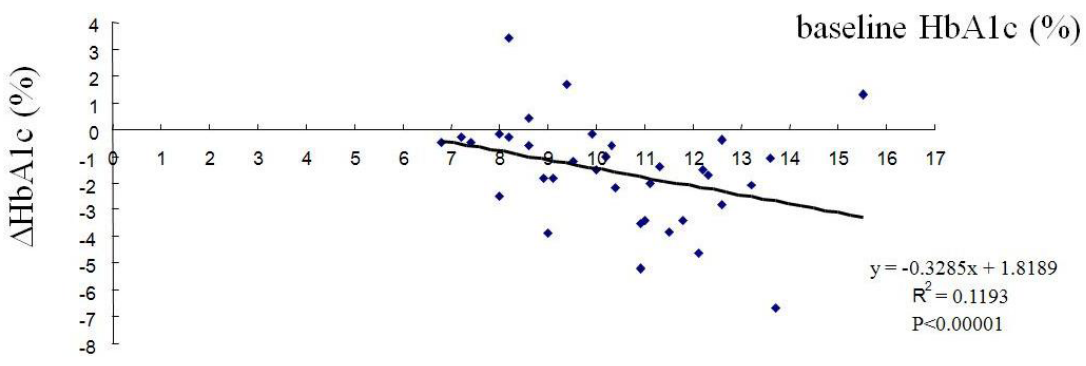

Figure 1. Correlations between the changes of $(\triangle \mathrm{HbA1c}$ and the baseline HbA1c levels. Simple regression analysis was performed between the indicated parameters.

A) sitagliptin B) alogliptin

with HOMA-R and BMI levels in both groups. By contrast HOMA-B levels significantly increased in both groups (see Figure $2,+91.2 \%$ increase for sitagliptin and $+52.3 \%$ increase for alogliptin) with significant inter-group differences $(p<0.05)$.

Next, one interesting question is whether the reductions $(\Delta)$ in glucose levels have any correlations with the changes $(\Delta)$ of beta-cell function (assessed by HOMA-B). For this purpose, simple regression analysis was performed using these parameters. Both in the sitagliptin and alogliptin groups, strong negative correlations were observed between $\triangle \mathrm{HbA} 1 \mathrm{C}$ and $\triangle \mathrm{HOMA}-\mathrm{B}(\mathrm{R}=-0.638$ for sitagliptin and $\mathrm{R}=-$ 0.565 for alogliptin, figure $3 A$ and $B$ ).

\section{Differential effects on lipid profiles between sitagliptin and alogliptin}

Effects on DPP-4 inhibitors on other metabolic parameters, for example, lipid profiles, remain largely uncharacterized. As an initial step towards investigating this question, a number of lipid parameters including T-C, TG, HDL, non$\mathrm{HDL}-\mathrm{C}, \mathrm{LDL}-\mathrm{C}$ and FFA were monitored.

In the sitagliptin group (Table IIA1), TG levels had a tendency to decrease $(-15.4 \%, p=0.0651)$ and FFA levels significantly decreased $(-15.3 \%, p<0.05)$. Then, baseline TG levels were divided into two groups; those with above
( $\geq$ )200mg/dl (high TG group) and others with below ( $\leq$ ) 199 $\mathrm{mg} / \mathrm{dl}$ (normal TG group). While no changes were observed in the normal TG group, significant reductions of TG levels were observed in the high TG group (-32.7\%, Table IIA2). No correlations were observed between the changes of $\mathrm{HbA1c}$ levels and those of TG or FFA (results not shown). In contrast to this, no changes of T-C, non-HDL, or LDL-C levels were observed (Table IIA1). In the alogliptin group (Table IIB), T-C, non-HDL and LDL-C levels significantly decreased while TG or FFA levels had no change. Interestingly, weakmoderate correlations were observed between the changes of $(\Delta) \mathrm{HbA} 1 \mathrm{c}$ and $\Delta \mathrm{T}-\mathrm{C}(\mathrm{R}=0.265), \Delta$ non-HDL-C $(\mathrm{R}=0.301)$ or $\Delta \mathrm{LDL}-\mathrm{C}(\mathrm{R}=0.339)$ in the alogliptin group as shown in Table III. No changes in HDL-C levels were observed in either sitagliptin or alogliptin group (Table IIA1 and IIB). Blood pressure was also monitored. However, the variations were so large and no conclusions have been made regarding the effect of sitagliptin or alogliptin on blood pressure (results not shown).

\section{Safety and tolerability}

4 out of 37 subjects in the sitagliptin group and 3 out of 35 subjects in the alogliptin group reported mid hypoglycemic events, which could be easily managed by taking glucose drinks or sweet candies by themselves. In most cases, this 


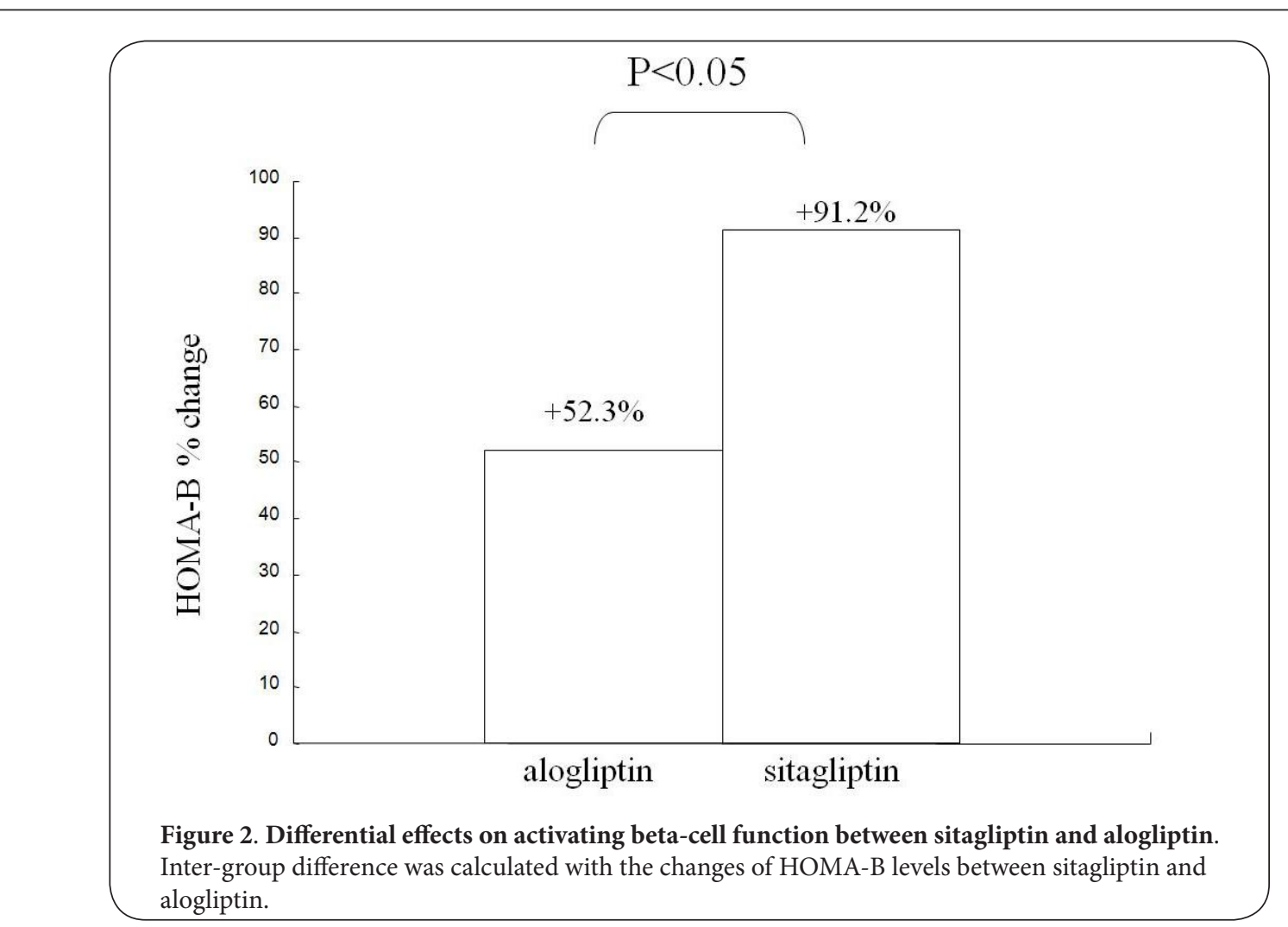

A: sitagliptin

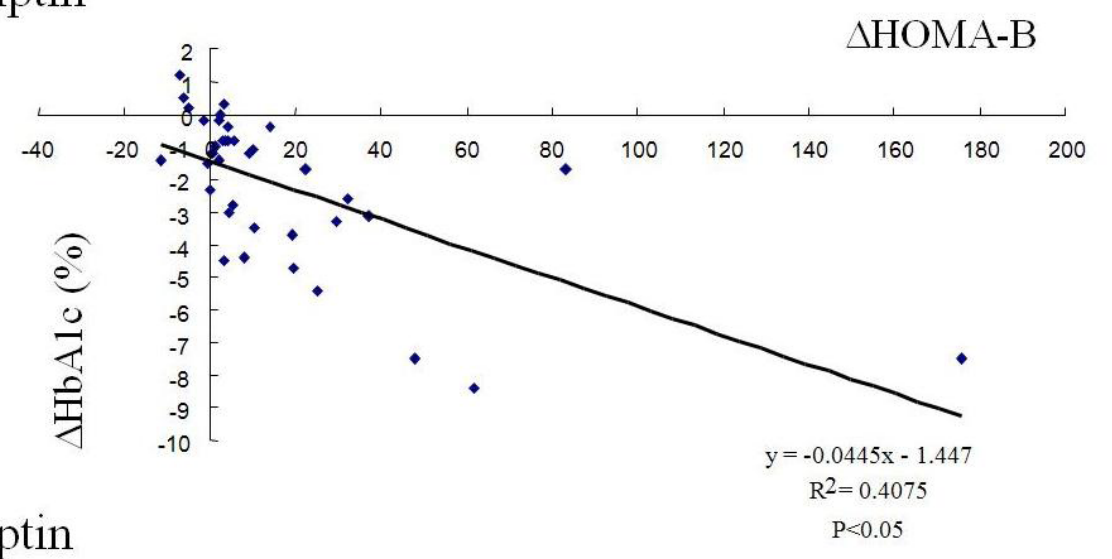

B: alogliptin

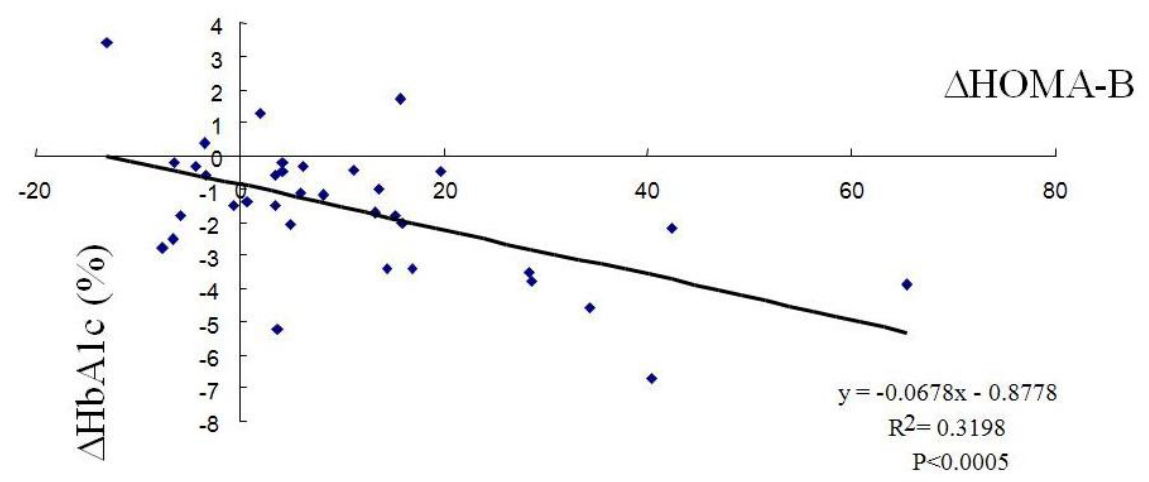

Figure 3. Correlations between the changes of $(\Delta) \mathrm{HbA1c}$ and $\Delta$ HOMA-B

Simple regression analysis was performed between the indicated parameters 
adverse event occurred within the first 2 weeks of the initiation of these drugs. Otherwise in both groups, no subjects had any clinically significant elevations of renal or hepatic enzymes and no gastrointestinal complains were observed. No subject had dropped out because of intolerance or adverse events. However, UA levels significantly increased in both groups (Table IIA1 and IIB).

\section{Discussion}

\section{Glycemic effects and safety}

In this present work, both sitagliptin $25-100 \mathrm{mg} /$ day and alogliptin $6.25-25 \mathrm{mg} /$ day monotherapy in newly diagnosed, drug naïve Japanese subjects with T2DM were shown to be rather effective and safe. However, around $10 \%$ subjects in both groups reported mild (not serious) hypoglycemic events. In addition, significant increases of UA levels were observed in both groups, though still within normal range (Table IIA1 and IIB). The clinical significance of the increased UA levels remains to be investigated.

In both groups, approximately $35 \%$ subjects were nonresponders whose $\mathrm{HbA1c}$ levels had less than $1 \%$ decrease in 3 months period. Currently we are investigating the clinical characterizations between responders and nonresponders with these drugs. In analogy to other oral hypoglycemic agents (OHAs), the response to sitagliptin or alogliptin is proportional to the baseline $\mathrm{HbA1c}$ levels (Figure 1). In this connection, both sitagliptin and alogliptin are rather effective with poorly controlled drug naïve subjects (HbA1c above 10\%). Although the numbers of the subjects in this study are small and the study duration is short, these results may implicate that both sitagliptin and alogliptin may be used as one of the first-line drugs for T2DM.

\section{Differential effects on beta-cell function}

Both sitagliptin and alogliptin were able to enhance betacell function as measured by the increase of HOMA-B levels, however, sitagliptin had a higher degree of upregulating this parameter (Figure 2). The reductions of $(\Delta)$ $\mathrm{HbA1c}$ levels had strong, negative correlations with the increases of $(\triangle)$ HOMA-B levels in both groups, however, the degrees of correlations might be somewhat different between sitagliptin $(R=-0.638)$ and alogliptin $(R=-0.565)$. These results indicate that glycemic efficacy of sitagliptin or alogliptin is obtained through activating beta-cell function, although with distinct degrees. So why do these two drugs have similar glucose lowering effects in spite of the fact that sitagliptin has a higher degree of enhancing beta-cell activity? One potential explanation for this question is the existence of GLP-1 independent pathway of DPP-4 inhibitors as has been hypothesized [23]. In that paper, it was proposed that the glycemic efficacy of DPP-4 inhibitors were medicated by not only through GLP-1 dependent pathway but also by other GLP-1 independent pathways (beyond inhibition of
DPP-4). Accordingly, the balance of dependency of DPP-4 inhibitors between these pathways (GLP-1 dependent and independent) may differ among different DPP-4 inhibitors; e.g. alogliptin has a higher degree of enhancing this GLP-1 independent pathway than sitagliptin. Further studies using molecular and cellular approaches will be necessary to investigate the mechanisms as questioned above.

\section{Differential effects on lipid profiles}

Effects on non-glycemic parameters, for example, lipid profiles, with DPP-4 inhibitors are inconsistent [16]. One of the novel findings in this work is that aloglitin could significantly reduce T-C, non-HDL-C or LDL-C levels, while sitagliptin could reduce high TG and FFA levels. Neither drug had effects on HDL-C levels (Table IIA1 and IIB). These effects differ from those of pioglitazone, a TZD class of insulin sensitizer which significantly reduced TG and increased $\mathrm{HDL}-\mathrm{C}$ levels while it had inconsistent effects on $\mathrm{T}-\mathrm{C}$ or LDL-C levels [21]. In this respect, combination of alogliptin and pioglitazone may be beneficial in controlling the overall lipid profiles in patients with T2DM. Anyway, the pharmacological efficacy of alogliptin to ameliorate these atherogenic cholesterols is an advantage and this drug may reduce the risks of cardiovascular disorders. Indeed, potent antiatherosclerotic effects of alogliptin in addition to its potent antidiabetic effects are recently speculated $[4,17,24]$. One interesting observation in this study is that the changes of $\mathrm{HbA} 1 \mathrm{c}$ levels were weakly correlated with those of T-C, non-HDL-C or LDL-C in the alogliptin group (Table III). At the present time, it remains to be investigated whether the changes of these atherogenic lipids are the cause or the consequence of the reduced glucose levels by alogliptin. Anyhow, these atherogenic lipids may be an independent marker for assessing the glycemic efficacy of alogliptin. At the same time, the molecular and cellular mechanisms of how these drugs lower lipid levels (e.g. effects on synthesis, secretion, absorption or clearance) will be an interesting research topic.

To this end, it is of interest to monitor the effect of other DPP-4 inhibitors (e.g. vildagliptin, saxagliptin) on lipid profiles. Although the mechanism of the action and the glycemic efficacy are comparable, these DPP-4 inhibitors show significant structural differences $[5,6]$. Several clinical trials of newer generations of DPP-4 inhibitors are currently ongoing. These results will help identify differences among these compounds.

Table III. Correlations between the changes of $(\triangle \mathrm{HbAlc}$ and those of lipid parameters in the alogliptin group.

Simple regression analysis was performed between the indicated lipid parameters.

$\triangle \mathrm{HbA} 1$ vs. $\triangle \mathrm{T}-\mathrm{C} \quad \mathrm{R}=0.265$

$\triangle$ HbA1c vs. $\triangle$ nonHDL-C $\quad \mathrm{R}=0.301$

$\triangle$ HbA1c vs. $\triangle \mathrm{LDL}-\mathrm{C} \quad \mathrm{R}=0.339$ 


\section{The limitations and strengthens of the study}

The limitations of this study are that the number of the subjects is small and the study duration is short. Furthermore, it is not strictly randomized. However one can assume that the observed changes were caused exclusively by these drugs (sitagliptin or alogliptin) based on the design of the study (monotherapy with drug naïve patients). Further randomized, double-blind, placebo-controlled longer period study with increased number of subjects will be necessary to strengthen the finding in this study.

\section{Conclusions}

This study suggests that both sitagliptin and alogliptin are effective and safe as an initial therapy for T2DM. However differential effects are observed on nonglycemic parameters including lipid and beta-cell function. Accordingly, the choice of sitagliptin or alogliptin (or other $\mathrm{OHAs}$ ) is dependent on the characteristics of the patients. For example, sitagliptin is suitable for those with high TG and/or FFA levels while alogliptin is useful for those with high atherogenic cholesterols (e.g. LDL-C and non-HDL-C). Further sitagliptin can be preferentially used in those with low beta-cell function (low insulin secretory capacity).

\section{Competing interests}

The authors declare that they have no competing interests. Acknowledgements

The authors thank Drs. Jan Wajs, Takashi Suzuki, Susumu Touchika, and Hiroshi Kawashima for discussions and Naoki Takeda for the advice of statistical analysis.

Publication history

Received: 26-Aug-2012 Revised: 30-Sep-2012

Accepted: 15-Oct-2012 Published: 05-Nov-2012

\section{References}

1. Lovshin JA, Drucker DJ: Incretin-based therapies for type 2 diabetes mellitus. Nat Rev Endocrinol 2009, 5:262-269. | Article| PubMed

2. Russell-Jones D, Gough S: Recent advances in incretin-based therapies. Clin Endocrinol (Oxf) 2012, 77:489-499. | Article| PubMed

3. Neumiller JJ: Clinical pharmacology of incretin therapies for type 2 diabetes mellitus: implications for treatment. Clin Ther 2011, 33:528-576. | Article| PubMed

4. Kutoh E, Ukai Y. Kutoh E, Ukai Y: Alogliptin as an initial therapy in patients with newly diagnosed, drug naive type 2 diabetes: a randomized, control trial. Endocrine 2012, 41:435-441. | Article| PubMed

5. Neumiller JJ: Differential chemistry (structure), mechanism of action, and pharmacology of GLP-1 receptor agonists and DPP-4 inhibitors. J Am Pharm Assoc (2003) 2009, 49 Suppl 1:S16-29. | PubMed

6. Baetta R, Corsini A: Pharmacology of dipeptidyl peptidase-4 inhibitors: similarities and differences. Drugs 2011, 71:1441-1467. | Articlel PubMed

7. Golightly LK, Drayna CC, McDermott MT: Comparative clinical pharmacokinetics of dipeptidyl peptidase-4 inhibitors. Clin Pharmacokinet 2012, 51:501-514. | Article| PubMed

8. Mohan V, Yang W, Son HY, Xu L, Noble L, Langdon RB, Amatruda $J M$, Stein PP, Kaufman KD: Efficacy and safety of sitagliptin in the treatment of patients with type 2 diabetes in China, India, and Korea. Diabetes Res Clin Pract 2009, 83:106-116. | Article
9. White J: Efficacy and safety of incretin based therapies: clinical trial data. J Am Pharm Assoc (2003) 2009, 49 Suppl 1:S30-40. | PubMed

10. Scheen AJ: DPP-4 inhibitors in the management of type 2 diabetes: a critical review of head-to-head trials. Diabetes Metab 2012, 38:89101. | Article| PubMed

11. Scott LJ: Alogliptin: a review of its use in the management of type 2 diabetes mellitus. Drugs 2010, 70:2051-2072. | Article| PubMed

12. White JR: Alogliptin for the treatment of type 2 diabetes. Drugs Today (Barc) 2011, 47:99-107. | Article| PubMed

13. Rendell M, Drincic A, Andukuri R: Alogliptin benzoate for the treatment of type 2 diabetes. Expert Opin Pharmacother 2012, 13:553-563. | Article| PubMed

14. DeFronzo RA, Fleck PR, Wilson CA, Mekki Q: Efficacy and safety of the dipeptidyl peptidase-4 inhibitor alogliptin in patients with type 2 diabetes and inadequate glycemic control: a randomized, doubleblind, placebo-controlled study. Diabetes Care 2008, 31:2315-2317. | Article| PubMed Abstract | PubMed Full Text

15. Takeuchi K, Fujita T, Hiroi S: [Pharmacological and clinical profile of alogliptin benzoate (NESINA(R))]. Nihon Yakurigaku Zasshi 2011, 137:43-50. | Article| PubMed

16. Monami M, Lamanna C, Desideri CM, Mannucci E: DPP-4 inhibitors and lipids: systematic review and meta-analysis. Adv Ther 2012, 29:14-25. | Article| PubMed

17. Eliasson B, Moller-Goede D, Eeg-Olofsson K, Wilson C, Cederholm J, Fleck P, Diamant M, Taskinen MR, Smith U: Lowering of postprandial lipids in individuals with type 2 diabetes treated with alogliptin and/or pioglitazone: a randomised double-blind placebo-controlled study. Diabetologia 2012, 55:915-925. | Article| PubMed

18. Tominaga M: [Diagnostic criteria for diabetes mellitus]. Rinsho Byori 1999, 47:901-908. | PubMed

19. Ma Y, Olendzki BC, Merriam PA, Chiriboga DE, Culver AL, Li W, Hebert $J R$, Ockene IS, Griffith JA, Pagoto SL: A randomized clinical trial comparing low-glycemic index versus ADA dietary education among individuals with type 2 diabetes. Nutrition 2008, 24:45-56. | Article| PubMed Abstract | PubMed Full Text

20. Tominaga M, Makino H, Yoshino G, Kuwa K, Takei I, Aono Y, Hoshino T, Umemoto M, Shimatsu A, Sanke T, Kuwashima M, Taminato T, Ono $\mathrm{J}$ : Japanese standard reference material JDS Lot 2 for haemoglobin A1c. II: Present state of standardization of haemoglobin A1c in Japan using the new reference material in routine clinical assays. Ann Clin Biochem 2005, 42:47-50. I Article| PubMed

21. Kutoh E: Differential regulations of lipid profiles between Japanese responders and nonresponders treated with pioglitazone. Postgrad Med 2011, 123:45-52. | Article| PubMed

22. Matthews DR, Hosker JP, Rudenski AS, Naylor BA, Treacher DF, Turner RC: Homeostasis model assessment: insulin resistance and beta-cell function from fasting plasma glucose and insulin concentrations in man. Diabetologia 1985, 28:412-419. | Article| PubMed

23. Kutoh E: Sitagliptin is effective and safe as add-on to insulin in patients with absolute insulin deficiency: a case series. J Med Case Rep 2011, 5:117. | Article| PubMed Abstract | PubMed Full Text

24. Kapoor S: Potent antiatherosclerotic effects of alogliptin in addition to its potent antidiabetic effects. Diabetes Metab Syndr Obes 2012, 5:121-123. | Article| PubMed Abstract | PubMed Full Text 